\title{
A nearly optimal preconditioner for the Navier-Stokes equations
}

\author{
Lina Hemmingsson-Frändén* Andrew Wathen ${ }^{\dagger}$
}

\begin{abstract}
We present a preconditioner for the linearised Navier-Stokes equations which is based on the combination of a fast transform approximation of an advection diffusion problem together with the recently introduced ' $B F B^{T}$ ', preconditioner of Elman (SIAM J. Sci. Comput. 20, pp. 1299-1316, 1999). The resulting preconditioner when combined with an appropriate Krylov subspace iteration method yields the solution in a number of iterations which appears to be independent of the Reynolds number provided a mesh Péclet number restriction holds, and depends only mildly on the mesh size. The preconditioner is particularly appropriate for problems involving a primary flow direction.
\end{abstract}

\section{Introduction}

Because of their wide applicability, there is considerable interest in effective and efficient solution algorithms for the steady incompressible Navier-Stokes equations

$$
\begin{aligned}
-\nu \Delta \mathbf{u}+(\mathbf{u} \cdot \nabla) \mathbf{u}+\nabla p & =\mathbf{f}, \quad \text { in } \Omega \subset \mathbb{R}^{d} . \\
-\nabla \cdot \mathbf{u} & =0, \quad \text {. }
\end{aligned}
$$

which must be posed together with appropriate boundary conditions. In this primitive variable formulation, the vector $\mathbf{u}$ is the fluid velocity, and the scalar $p$ is the pressure (which is defind only up to an additive constant), and we may be interested in 2- or 3-dimensional problems $(d=2$ or 3$)$. A straightforward linearisation of the quadratically nonlinear convection term in the form $\mathbf{u}^{(i-1)} \cdot \nabla \mathbf{u}^{(i)}$ leads to a Picard iteration where each iterative step involves the solution of an Oseen problem

$$
\begin{aligned}
-\nu \Delta \mathbf{u}+(\mathbf{w} \cdot \nabla) \mathbf{u}+\nabla p & =\mathbf{f}, \quad \text { in } \Omega \subset \mathbb{R}^{d} . \\
-\nabla \cdot \mathbf{u} & =0, \quad \text {. }
\end{aligned}
$$

* Oxford University Computing Laboratory, Wolfson Building, Parks Road, Oxford OX1 3QD, UK and Department of Information Technology, Uppsala University, Sweden, this author was supported by the Swedish Research Council for Engineering Sciences under contract No. 282-951000 UK

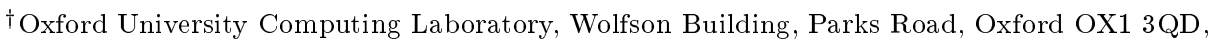


where the vector $\mathbf{w}$ is known. It is these equations with which we are concerned in this paper. Convergence of the Picard iteration in this situation is proved in [15]. We will take simple Dirichlet boundary conditions of the form $\mathbf{u}=\mathbf{g}$ on $\partial \Omega$ for the velocity, though the techniques we present could apply more generally.

The important parameter in (1) is the 'viscosity' parameter $\nu$ (the inverse of the Reynolds number). For larger values of $\nu$, the problem becomes dominated by the viscous diffusion and the related (linear and self-adjoint) Stokes problem

$$
\begin{aligned}
-\nu \Delta \mathbf{u}+\nabla p & =\mathbf{f}, \quad \text { in } \Omega \subset \mathbb{R}^{d}, \\
-\nabla \cdot \mathbf{u} & =0,
\end{aligned}
$$

becomes an appropriate approximation in many instances. There has been much work on efficient solution methods for approximations of the Stokes problem: a number of optimal iterative techniques are compared by Elman [4]. For smaller values of $\nu$ the advection term in (1) becomes dominant: we are here interested in techniques which apply over the whole range of values of $\nu(>0)$.

Discretisation of (1) (or (2)) yields linear systems of the form

$$
\left(\begin{array}{cc}
F & B^{T} \\
B & 0
\end{array}\right)\left(\begin{array}{l}
\mathbf{u} \\
\mathbf{p}
\end{array}\right)=\left(\begin{array}{l}
\mathbf{f} \\
0
\end{array}\right),
$$

where $\mathbf{u} \in \mathbb{R}^{n}$ is a vector of approximate values of the velocity components and $\mathbf{p} \in \mathbb{R}^{m}$ is a vector of discrete pressures. The square matrix $F$ is a discrete advectiondiffusion operator which we write as $\nu A+N$ where $A$ is the discrete vector Laplacian coming from the viscous term $\Delta \mathbf{u}$ and $N$ is the discrete representation of the advection $\mathbf{w} \cdot \nabla \mathbf{u}$. In order to mimic the underlying differential operators, there is usually good reason for the discrete gradient $B^{T}$ (which applies to the pressures) to be the adjoint of the discrete (negative) divergence $B$ as given here.

It is well known that issues of LBB- (or pressure-) stability arise in approximations of the Stokes and Oseen equations: see for example [1] for mixed finite element approximation. In the case of finite difference approximation practitioners found the device of employing staggered grids ([11]) to avoid spurious pressure modes and this has more recently been underpinned by proof of pressure stability for the popular maker in cell (MAC) scheme [14]. For spectral approximation the situation is slightly more complicated [23]. We will merely assume that an appropriate LBB-stable approximation has been used to yield the system (3), the solution of which is the subject matter of this paper.

Direct solution of (3) is a feasible option for relatively coarse grid discretisations (see e.g. Duff, Erisman and Reid[3]), but iterative methods are required for larger discrete problem sizes. A number of iterative approaches have been proposed. Solvers of pressure-correction type have been used for the last 20 or more years (see e.g. [19]). These are based on an implicit elimination of the velocity variables and iterative solution of the resulting Schur complement system

$$
B F^{-1} B^{T} \mathbf{p}=B F^{-1} \mathbf{f}
$$

usually employing an approximation of $F^{-1}$ and a consequent inner iteration (see for example [20]). More recently multigrid and preconditioned Krylov subspace 
methods have been used: see [4] for a description and comparison of competing approaches of these types.

If $h$ represents a discrete mesh size, then a desirable property of any iterative solver is independence (or mild dependence) of the convergence with respect to $h$ as $h \rightarrow 0$. For sparse discretisations an amount of work per iteration dependent only linearly on the size of the discrete problem can usually easily be ensured. In the Oseen problem, independence of the convergence rate with respect to $\nu$ (as $\nu \rightarrow 0$ ) is also very desirable. Iterative solvers which have $h$-independent convergence but for which convergence deteriorates for small $\nu$ are described in [6] and [9]. These solvers are effective for low Reynolds number flows, but the desire for rapidly converging methods also for small $\nu$ recently lead Elman [5] to suggest his so-called ' $B F B^{T}$ '-preconditioner which can be employed together with any nonsymmetric iterative algorithm such as GMRES [21], BiCGSTAB [24] or QMR [8]. Elman's preconditioner employed with GMRES converges at rates which are apparently independent of $\nu$ and only mildly dependent on $h$. (though this is not so far rigorously proved). The method therefore gives a very attractive solution algorithm for Oseen problems. The most significant remaining practical issue is that the $B F B^{T}$-preconditioner requires the solution or approximation of an associated advection-diffusion subproblem involving $F$ as coefficient matrix.

In this paper we present a number of related variants of Elman's $B F B^{T}$ preconditioner which are based on fast transform approximations of the advection-diffusion operator $F$, which have recently been worked on by the first author [12]. There it is shown that use of these semi-circulant matrices as preconditioners for advectiondiffusion problems leads to almost optimal solution algorithms (with computation complexity $\mathcal{O}\left(N \log _{2} N\right)$ for a discrete problem of size $\left.N\right)$ for uni-directional advection.

In this paper we show that the combination of Elman's $B F B^{T}$ preconditioner employing Hemmingsson's semi-circulant approximation for the advection-diffusion subproblem yields highly effective solution algorithms for the Oseen problem. These practical algorithms display convergence which is appearently independent of $\nu$ and only mildly dependent on $h$.

In Section 2 we describe Elman's $B F B^{T}$ preconditioner for the Oseen problem and the fast transform methods of Hemmingsson for advection-diffusion and their combination. In Section 3 we describe the discretisation used, and in Section 4 we give some guiding theoretical analysis which indicates why our new method might be efficient modifications of Elman's preconditioner. Computational results employing the MAC finite difference scheme both for uni-directional flow and flow over a backwards facing step are given in Section 5. Our conclusions are reserved for Section 6. 


\section{$2 B F B^{T}$ and semi-circulant preconditioner}

Based on work of Silvester and Wathen [22], and Elman and Silvester [6] (see [7] for an overview), Elman [5] considered preconditioning (3) with a matrix of the form

$$
\left(\begin{array}{cc}
F & B^{T} \\
0 & X
\end{array}\right)
$$

The same motivation lead Murphy and Wathen [18] (see also [17]) to consider the associated diagonal preconditioner

$$
\left(\begin{array}{ll}
F & 0 \\
0 & X
\end{array}\right)
$$

In either approach, the matrix $X$ would ideally be chosen to approximate the Schur complement $B F^{-1} B^{T}$, see [5]. Elman shows that for various discretisation schemes (including the MAC method) which satisfy a projection property, the choice

$$
X^{-1}=\left(B B^{T}\right)^{-1}\left(B F B^{T}\right)\left(B B^{T}\right)^{-1}
$$

accurately approximates the inverse of the Schur complement and thus renders (5) a good preconditioner. Note multiplication by $X^{-1}$ is required in the preconditioner step. The operators indicated by $\left(B B^{T}\right)^{-1}$ are readily replaced by a simple multigrid procedure (see section 4 ) since $B B^{T}$ is a discrete Laplace operator on the pressures $([10])$. Indeed a crude single multigrid V-cycle is effective since exact solution in the preconditioning block matrix $X$ is not required and multigrid exhibits spectral equivalence for the (symmetric) Laplacian problem. The $B F B^{T}$ part of the preconditioner simply involves multiplication.

The application of (5) now requires inversion of $F$. Elman demonstrates that this might be achieved by an inner iteration and presents results with Gauss-Seidel sweeping. Here we employ a semi-circulant approximation to $F$.

We will assume a problem in two space dimensions. The matrix $F$ is then of the form

$$
F=\left(\begin{array}{cc}
F^{(1)} & 0 \\
0 & F^{(2)}
\end{array}\right)
$$

where $F^{(i)}=\nu A^{(i)}+N^{(i)}, i=1,2$. We will consider all types of discretisations on an $m_{1} \times m_{2}$-grid, yielding a general $F^{(i)}$ as

$$
F^{(i)}=\left(\begin{array}{cccc}
{\left[F^{(i)}\right]_{1,1}} & {\left[F^{(i)}\right]_{1,2}} & \cdots & {\left[F^{(i)}\right]_{1, m_{2}}} \\
{\left[F^{(i)}\right]_{2,1}} & \ddots & \ddots & \vdots \\
\vdots & \ddots & \ddots & {\left[F^{(i)}\right]_{m_{2}-1, m_{2}}} \\
{\left[F^{(i)}\right]_{m_{2}, 1}} & \cdots & {\left[F^{(i)}\right]_{m_{2}, m_{2}-1}} & {\left[F^{(i)}\right]_{m_{2}, m_{2}}}
\end{array}\right)
$$


Now define a general circulant matrix $C$ of order $m_{1}$ by

$$
C=\left(\begin{array}{cccc}
c_{1} & c_{2} & \cdots & c_{m_{1}} \\
c_{m_{1}} & \ddots & \ddots & \vdots \\
\vdots & \ddots & \ddots & c_{2} \\
c_{2} & \cdots & c_{m_{1}} & c_{1}
\end{array}\right)
$$

It is well-known (see [16]) that a circulant matrix has an eigen-decomposition in Fourier-matrices:

$$
C=\mathcal{F}_{m_{1}} \Lambda \mathcal{F}_{m_{1}}^{H}
$$

where

$$
\begin{gathered}
\mathcal{F}_{m_{1}}(k, j)=\frac{1}{\sqrt{m_{1}}} \exp \left(i 2 \pi(j-1)(k-1) / m_{1}\right) \quad, \quad k, j=1, \ldots, m_{1}, \\
\Lambda=\operatorname{diag}\left(\lambda_{1}, \ldots, \lambda_{m_{1}}\right)
\end{gathered}
$$

and

$$
\lambda_{k}=\sum_{j=1}^{m_{1}} c_{j} \exp \left(i 2 \pi(j-1)(k-1) / m_{1}\right) \quad, \quad k=1, \ldots, m_{1} .
$$

The system of equations $C x=y$ can hence be solved by the fast Fourier transform algorithm (FFT), the solution of a diagonal system and an inverse fast Fourier transform (IFFT).

In [2] it is shown that the optimal circulant approximation $C$ to a general matrix $B$ of order $m_{1}$, in the sense that the following quantity is minimized

$$
\|C-B\|_{F},
$$

where $\|\cdot\|_{F}$ denotes the Frobenius norm, is obtained from

$$
c_{\ell}=\frac{1}{m_{1}} \sum_{k=1}^{m_{1}} B(k, j) \quad, \quad j=(k+\ell-2) \bmod \left(m_{1}\right)+1 .
$$

In [13] this result is generalized to block-matrices of the type defined in (7). Define a semi-circulant matrix of order $m_{1} \times m_{2}$ by

$$
\mathcal{C}=\left(\begin{array}{cccc}
{[\mathcal{C}]_{1,1}} & {[\mathcal{C}]_{1,2}} & \ldots & {[\mathcal{C}]_{1, m_{2}}} \\
{[\mathcal{C}]_{2,1}} & \ddots & \ddots & \vdots \\
\vdots & \ddots & \ddots & {[\mathcal{C}]_{m_{2}-1, m_{2}}} \\
{[\mathcal{C}]_{m_{2}, 1}} & \cdots & {[\mathcal{C}]_{m_{2}, m_{2}-1}} & {[\mathcal{C}]_{m_{2}, m_{2}}}
\end{array}\right)
$$

where $[\mathcal{C}]_{k, j}$ are circulant matrices. From (8) it follows that

$$
\mathcal{C}=\left(I_{m_{2}} \otimes \mathcal{F}_{m_{1}}\right) \mathcal{T}\left(I_{m_{2}} \otimes \mathcal{F}_{m_{1}}\right)^{H},
$$


where $\otimes$ denotes the Kronecker-product and $\mathcal{T}$ has blocks that are all diagonal. Thus the solution of

$$
\mathcal{T} x=y
$$

decouples into the solution of $m_{1}$ systems of equations of order $m_{2}$. If the discretisation used is narrow, these systems of equations are narrow-banded. In [13] it is shown that the optimal semi-circulant approximation $\mathcal{C}$ to a general block-matrix $\mathcal{B}$ is obtained from

$$
\begin{gathered}
{[\mathcal{C}]_{k, j}=\left(\begin{array}{cccc}
c_{1} & c_{2} & \cdots & c_{m_{1}} \\
c_{m_{1}} & \ddots & \ddots & \vdots \\
\vdots & \ddots & \ddots & c_{2} \\
c_{2} & \cdots & c_{m_{1}} & c_{1}
\end{array}\right),} \\
c_{\ell}=\frac{1}{m_{1}} \sum_{\bar{k}=1}^{m_{1}}[\mathcal{B}]_{k, j}(\bar{k}, \bar{\jmath}) \quad, \quad \bar{\jmath}=(\bar{k}+\ell-2) \bmod \left(m_{1}\right)+1 .
\end{gathered}
$$

Here optimality is again in the Frobenius-norm sense.

Thus we conclude that we can derive the optimal semi-circulant approximation $\tilde{F}^{(i)}$ to $F^{(i)}$ by using (10) and (11). Finally from (9) we get that

$$
\tilde{F}^{(i)} x=y
$$

can be solved by

- $m_{2}$ FFT:s of vectors of length $m_{1}$,

- the solution of $m_{1}$ systems of equations of order $m_{2}$,

- $m_{2}$ IFFT:s of vectors of length $m_{1}$.

Using symmetries it can be shown that the (I)FFT:s can be performed on vectors of length $m_{1} / 2$. Similarly only $m_{1} / 2$ of the intermediate systems of equations have to be solved for.

In this paper we will study two different preconditioners that both have their origin in the $B F B^{T}$-preconditioner defined by (5) and (6). They are both modified from the original preconditioner, here denoted by $\mathcal{Q}$, in such a way that $F$ is approximated by a semi-circulant matrix, hence allowing for Fourier techniques in the preconditioner solve. Denote by $\tilde{F}$ the semi-circulant approximation of $F$ defined by

$$
\tilde{F}=\left(\begin{array}{cl}
\tilde{F}^{(1)} & 0 \\
0 & \tilde{F}^{(2)}
\end{array}\right) .
$$

We then define the new preconditioners $\tilde{\mathcal{Q}}$ and $\tilde{\mathcal{Q}}$ by

$$
\tilde{\mathcal{Q}}=\left(\begin{array}{cc}
\tilde{F} & B^{T} \\
0 & X
\end{array}\right),
$$


and

$$
\tilde{\tilde{\mathcal{Q}}}=\left(\begin{array}{cc}
\tilde{F} & B^{T} \\
0 & \tilde{X}
\end{array}\right)
$$

where $X$ is defined by (6) and

$$
\tilde{X}^{-1}=\left(B B^{T}\right)^{-1}\left(B \tilde{F} B^{T}\right)\left(B B^{T}\right)^{-1} .
$$

Since $\tilde{\mathcal{Q}}^{-1}$ and $\tilde{\tilde{\mathcal{Q}}}^{-1}$ can be factorised as

$$
\tilde{\mathcal{Q}}^{-1} / \tilde{\tilde{\mathcal{Q}}}^{-1}=\left(\begin{array}{cc}
\tilde{F}^{-1} & 0 \\
0 & I
\end{array}\right)\left(\begin{array}{cc}
I & -B^{T} \\
0 & I
\end{array}\right)\left(\begin{array}{cc}
I & 0 \\
0 & X^{-1} / \tilde{X}^{-1}
\end{array}\right)
$$

we conclude that the preconditioner solve can be accomplished through:

- matrix-vector products,

- the direct solution of/a multi-grid procedure for the $B B^{T}$-systems,

- (I)FFT:s,

- the solution of (narrow-banded) systems of equations.

The system of equations

$$
\tilde{F} x=y
$$

is generally much less expensive to solve than

$$
F x=y
$$

with respect to computer memory and number of arithmetic operations. We will discuss this aspect further in Sections 3 and 5.

\section{Computational grid and discretisation}

We consider the MAC scheme on the staggered grid (see Figure 1) with space step $h_{i}$ in the $x_{i}$-direction, $i=1,2$. Let $\mathbf{u}=\left(\begin{array}{ll}u & v\end{array}\right)^{T}$ denote the unknown velocity field, and $\mathbf{w}=\left(\begin{array}{ll}a & b\end{array}\right)^{T}$ the wind. The discrete values of the pressure lie in the cell centers and the discrete values of $u$ and $v$ lie in the centers of the cell boundaries orthogonal to the $x_{1}$-axis and the $x_{2}$-axis respectively. 


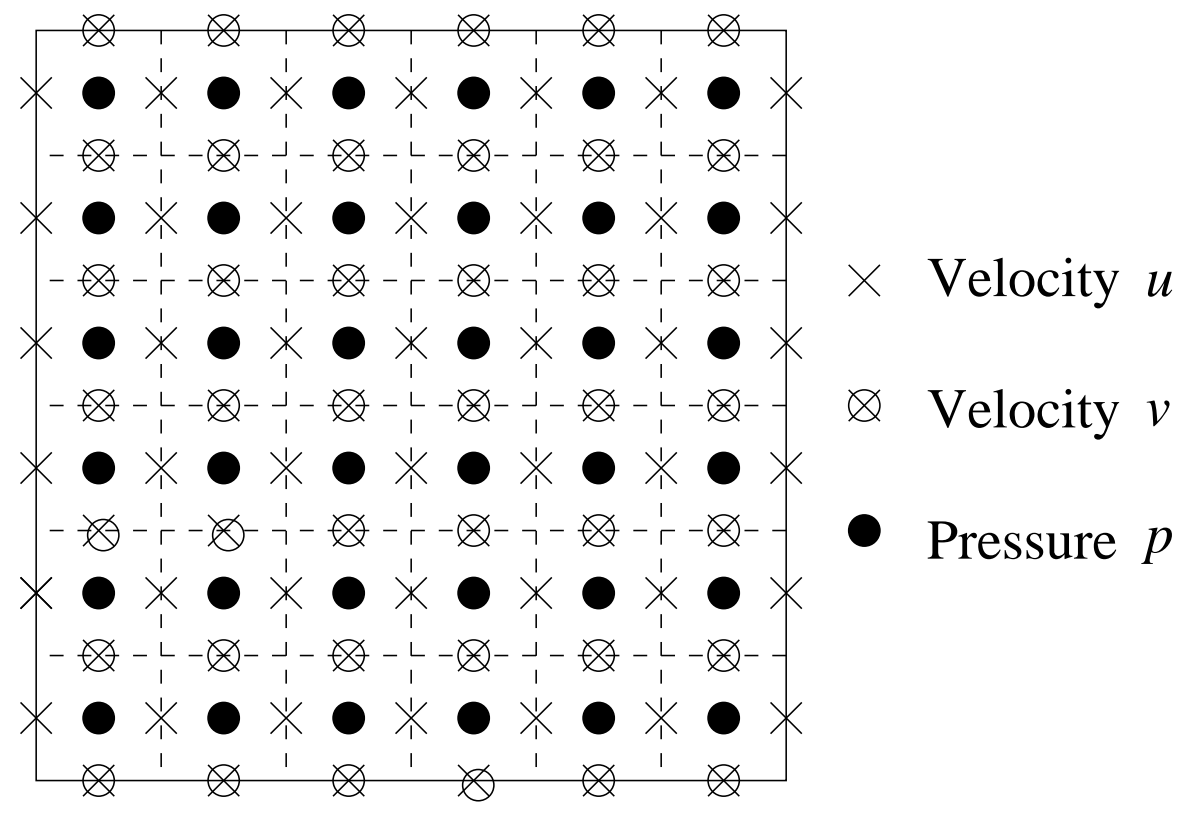

Figure 1: The staggered grid.

Denote by $u_{k, j}$ the discrete value of $u$ at the point $\left(k h_{1}, j h_{2}\right)$, and similarly for $v$ and $p$. The indices $(k, j)$ depend on the mesh function to which they correspond. In particular they need not be integers. The MAC scheme is then defined by

$$
\begin{aligned}
{[-\Delta u]_{k, j} } & \approx \frac{-u_{k+1, j}+2 u_{k, j}-u_{k-1, j}}{h_{1}^{2}}+\frac{-u_{k, j+1}+2 u_{k, j}-u_{k, j-1}}{h_{2}^{2}}, \\
{\left[a \frac{\partial u}{\partial x_{1}}\right]_{k, j} } & \approx \frac{a_{k+1 / 2, j} u_{k+1, j}-a_{k-1 / 2, j} u_{k-1, j}}{2 h_{1}} \\
{\left[b \frac{\partial u}{\partial x_{2}}\right]_{k, j} } & \approx \frac{b_{k, j+1 / 2} u_{k, j+1}-b_{k, j-1 / 2} u_{k, j-1}}{2 h_{2}} \\
{\left[\frac{\partial p}{\partial x}\right]_{k, j} } & \approx \frac{p_{k+1 / 2, j}-p_{k-1 / 2, j}}{h_{1}}
\end{aligned}
$$

and similarly for the derivatives associated with $v$.

The staggering of the grid imposes difficulties at the boundaries $x_{1}=0$ and $x_{1}=1$ for $u$, and at $x_{2}=0$ and $x_{2}=1$ for $v$. To prescribe the Dirichlet boundary conditions at those boundaries, we use linear extrapolation. For $u$ this gives

$$
\begin{aligned}
u_{-h_{1} / 2, j} & =-u_{h_{1} / 2, j}+2 u\left(0, x_{2, j}\right) \\
u_{1+h_{1} / 2, j} & =-u_{1-h_{1} / 2, j}+2 u\left(1, x_{2, j}\right) \quad, \quad j=1, \ldots, m_{2}
\end{aligned}
$$

and similarly for $v$. 
Consider a problem with $m=m_{1}=m_{2}$ gridpoints in each space-direction. If we use banded Gaussian elimination to solve (15), this requires $\simeq 8 m^{4}$ arithmetic operations (a.o.) in a factorisation phase, and $\simeq 12 \mathrm{~m}^{3}$ a.o. in a substitution phase. The solution of (14) on the other hand yields $\simeq 38 m^{2}$ a.o. and $\simeq 10 \mathrm{~m}^{2} \log _{2} m+78 \mathrm{~m}^{2}$ a.o. in the respective phases. In Section 5 we will discuss what these differences give, when we take the amount of iterations required into account.

\section{Theoretical analysis}

In [12] second-order centered differences are used to discretise the linearized advectiondiffusion equation

$$
-\nu \Delta u+(\mathbf{w} \cdot \nabla) u=f .
$$

A semi-circulant preconditioner for the resulting system of equations is analyzed theoretically with respect to location of eigenvalues and conditioning of the eigenvector matrix. Using these results, bounds on the asymptotic convergence rate can be obtained.

Define the mesh Péclet number $P e$ by $P e=\|w\|_{\infty} h / \nu$. In [12] it is shown that for mesh Péclet numbers less than 2, the rate of convergence depends only on the mesh Péclet number and the direction of the convective field and not on the spatial grid ratio nor the number of unknowns. The convergence rate improves with increasing mesh Péclet number for this scalar model problem. We can therefore hope that the only mild dependence on $h$ using the $B F B^{T}$-preconditioner is mainly retained, while the behaviour as $\nu \rightarrow 0$ for constant $h$ is in some sense even improved. This will be numerically examined in Section 5 .

Denote by $\mathcal{A}$ the coefficient matrix of (3). We will use right preconditioning and solve

$$
\mathcal{A} \mathcal{Q}^{-1} \mathbf{x}=\left(\begin{array}{l}
\mathbf{f} \\
0
\end{array}\right) \quad, \quad \mathcal{Q}\left(\begin{array}{l}
\mathbf{u} \\
\mathbf{p}
\end{array}\right)=\mathbf{x}
$$

Since

$$
\mathcal{A} \mathcal{Q}^{-1}=\left(\begin{array}{cc}
I & 0 \\
B F^{-1} & B F^{-1} B^{T} X^{-1}
\end{array}\right)
$$

the eigenvalues of $\mathcal{A} \mathcal{Q}^{-1}$ are

$$
\{1\} \cup \sigma\left(B F^{-1} B^{T} X^{-1}\right) .
$$

In [5] a problem on $\Omega=[0,1] \times[0,1]$ with constant wind $\mathbf{w}$ and periodic boundary conditions is analyzed. It is shown that for this case

$$
B F^{-1} B^{T} X^{-1}=I
$$

and hence all eigenvalues of (16) are equal to 1 . Since $B F^{-1} \neq 0$ in (16) it is clear that $\mathcal{A} \mathcal{Q}^{-1}-I \neq 0$, but $\left(\mathcal{A} \mathcal{Q}^{-1}-I\right)^{2}=0$ so that the minimum polynomial of $\mathcal{A} \mathcal{Q}^{-1}$ is of degree 2 and hence a Krylov subspace method will converge (terminate) in only 2 iterations.

For constant wind and periodic boundary conditions it is easily seen that $F=\tilde{F}$ and hence $\mathcal{Q}=\tilde{\mathcal{Q}}=\tilde{\tilde{\mathcal{Q}}}$. We summarize this result in a theorem. 
Theorem 4.1 Consider the Oseen problem defined by (1), discretised using the $M A C$ scheme. Then a Krylov subspace method using right preconditioning with $\mathcal{Q}$, $\tilde{\mathcal{Q}}$, or $\tilde{\tilde{\mathcal{Q}}}$ defined in (5), (12), (13), and (6) for the systems of equations (3) converges in only 2 iterations.

For non-constant wind and/or different boundary conditions this is no longer strictly true, but the number of iterations remains small.

\section{Computational results}

To start with, we made a number of experiments the same as those that appear in [5] in order to be able to compare our results with the original $B F B^{T}$-preconditioner $\mathcal{Q}$. The wind $\mathbf{w}$ is given by $\left(\begin{array}{ll}1 & 2\end{array}\right)^{T}$, and to obtain the right-hand side $\mathbf{f}$ in (3) we use a normally distributed random vector with mean 0 and variance 1 . The initial guess used is $\mathbf{u}^{0}=0$, and $p^{0}=0$, and the stopping criterion is

$$
\frac{\left\|\left(\begin{array}{l}
\mathbf{f} \\
0
\end{array}\right)-\left(\begin{array}{cc}
F & B^{T} \\
B & 0
\end{array}\right)\left(\begin{array}{l}
\mathbf{u} \\
\mathbf{p}
\end{array}\right)^{k}\right\|_{2}}{\left\|\left(\begin{array}{l}
\mathbf{f} \\
0
\end{array}\right)\right\|_{2}} \leq 10^{-6} .
$$

We have compared the results obtained with the original $B F B^{T}$-preconditioner with those obtained using semi-circulant approximations. Four variants of these approximations have been employed, $\tilde{\mathcal{Q}}_{x_{1}}, \tilde{\mathcal{Q}}_{x_{1} x_{2}}, \tilde{\tilde{\mathcal{Q}}}_{x_{1}}$, and $\tilde{\tilde{\mathcal{Q}}}_{x_{1} x_{2}}$. Here the subindex $x_{1}$ means that the circulant approximations are made along the $x_{1}$-axis both for $\tilde{F}^{(1)}$ and $\tilde{F}^{(2)}$, while the subindex $x_{1} x_{2}$ represents approximations along the $x_{1}$-axis for $\tilde{F}^{(1)}$ and along the $x_{2}$-axis for $\tilde{F}^{(2)}$.

In Table 1-5 we display the number of iterations obtained for varying $h=h_{1}=h_{2}$ and $\nu$. The lower left corner below the double bar indicates where the mesh Péclet number condition is violated. When we decrease $\nu$ for fixed $h$, the iteration counts remain low for a while but eventually increase as the mesh Péclet number condition is more severely violated: even divergence is seen. This is due to the fact that the results of [12] do not apply when the mesh Péclet number condition is violated and poorer convergence is evident.

\begin{tabular}{|c|c|c|c|}
\hline$h$ & $1 / 16$ & $1 / 32$ & $1 / 64$ \\
\hline$\nu=1$ & 9 & 10 & 12 \\
$\nu=1 / 10$ & 8 & 11 & 15 \\
$\nu=1 / 30$ & 9 & 10 & 13 \\
$\nu=1 / 50$ & 9 & 10 & 11 \\
$\nu=1 / 100$ & 10 & 12 & 11 \\
$\nu=1 / 200$ & 10 & 12 & 14 \\
\hline
\end{tabular}

Table 1: Number of iterations using $Q$. 


\begin{tabular}{|c|c|c|c|}
\hline$h$ & $1 / 16$ & $1 / 32$ & $1 / 64$ \\
\hline$\nu=1$ & 25 & 31 & 39 \\
$\nu=1 / 10$ & 25 & 30 & 39 \\
$\nu=1 / 30$ & 19 & 24 & 35 \\
$\nu=1 / 50$ & 21 & 21 & 30 \\
$\nu=1 / 100$ & 29 & 22 & 22 \\
$\nu=1 / 200$ & 37 & 32 & 25 \\
\hline
\end{tabular}

Table 2: Number of iterations using $\tilde{\mathcal{Q}}_{x_{1}}$.

\begin{tabular}{|c|c|c|c|}
\hline$h$ & $1 / 16$ & $1 / 32$ & $1 / 64$ \\
\hline$\nu=1$ & 20 & 24 & 31 \\
$\nu=1 / 10$ & 22 & 27 & 33 \\
$\nu=1 / 30$ & 24 & 24 & 30 \\
$\nu=1 / 50$ & 28 & 26 & 27 \\
$\nu=1 / 100$ & 29 & 24 & 22 \\
$\nu=1 / 200$ & 37 & 33 & 28 \\
\hline
\end{tabular}

Table 3: Number of iterations using $\tilde{\mathcal{Q}}_{x_{1} x_{2}}$.

\begin{tabular}{|c|c|c|c|}
\hline$h$ & $1 / 16$ & $1 / 32$ & $1 / 64$ \\
\hline$\nu=1$ & 28 & 34 & 42 \\
$\nu=1 / 10$ & 26 & 33 & 46 \\
$\nu=1 / 30$ & 19 & 25 & 40 \\
$\nu=1 / 50$ & 21 & 21 & 32 \\
$\nu=1 / 100$ & 39 & 35 & 33 \\
$\nu=1 / 200$ & div. & 50 & 40 \\
\hline
\end{tabular}

Table 4: Number of iterations using $\tilde{\tilde{\mathcal{Q}}}_{x_{1}}$.

\begin{tabular}{|c|c|c|c|}
\hline$h$ & $1 / 16$ & $1 / 32$ & $1 / 64$ \\
\hline$\nu=1$ & 23 & 29 & 36 \\
$\nu=1 / 10$ & 24 & 31 & 37 \\
$\nu=1 / 30$ & 26 & 28 & 35 \\
$\nu=1 / 50$ & 29 & 28 & 32 \\
$\nu=1 / 100$ & 40 & 34 & 28 \\
$\nu=1 / 200$ & div. & 55 & 36 \\
\hline
\end{tabular}

Table 5: Number of iterations using $\tilde{\tilde{\mathcal{Q}}}_{x_{1} x_{2}}$. 
From Tables $1-5$ we see that $\tilde{Q}$ yields fewer iterations than $\tilde{\widetilde{Q}}$. Moreover, the preconditioners with approximations made along different axis generally perform better than those with approximations made along the $x_{1}$-axis only. Thus $\tilde{Q}_{x_{1} x_{2}}$ seems to be the best choice among the semi-circulant $B F B^{T}$-preconditioners presented here. Note that the $x_{1}$-preconditioners seem to behave as we predicted in Section 4 ; the number of iterations decreases with decreasing $\nu$, and the dependence on $h$ is slightly stronger than for the original $B F B^{T}$-preconditioner.

Tables 1-2 show that the semi-circulant preconditioners clearly require more iterations than the original $B F B^{T}$-preconditioner. However, as we pointed out in Section 3, the solution of (15) needed in the original $B F B^{T}$-preconditioner requires more arithmetic operations than the solution of (14) used in the preconditioners presented here. In Figure 2 we display the quotients between the number of arithmetic operations required to solve (14) $\tilde{i t}$ times and the number of arithmetic operations to solve (15) it times. Here it denotes the number of iterations obtained with $\mathcal{Q}$ and $\tilde{i t}$ the number of iterations obtained with $\tilde{\mathcal{Q}}$ or $\tilde{\tilde{\mathcal{Q}}}$. We have accounted for one factorisation and it or $\tilde{i t}$ substitutions.
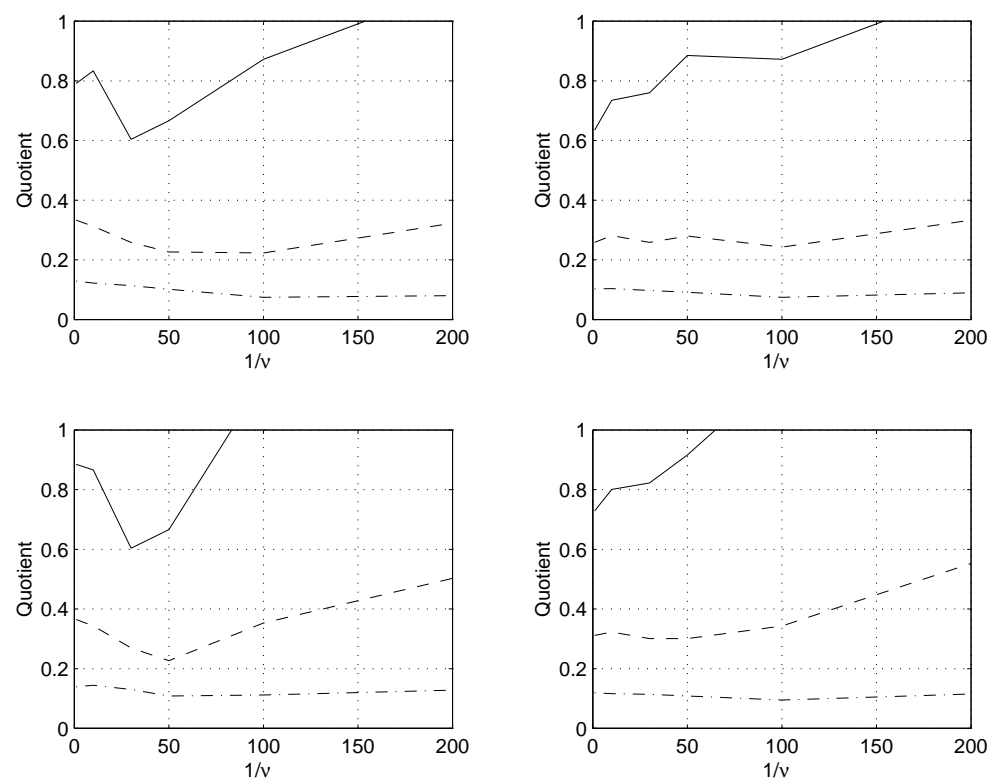

Figure 2: The quotients between the number of arithmetic operations required in the solution of (14) and (15) as a function of $1 / \nu$. A solid line represents a problem with $h=1 / 16$, a dashed line $h=1 / 32$, and a dashed-dotted line $h=1 / 64$. $\tilde{\mathcal{Q}}_{x_{1}}$ is displayed in top left, $\tilde{\mathcal{Q}}_{x_{1} x_{2}}$ in top right, $\tilde{\tilde{\mathcal{Q}}}_{x_{1}}$ in bottom left, and $\tilde{\tilde{\mathcal{Q}}}_{x_{1} x_{2}}$ in bottom right.

Figure 2 shows that using a semi-circulant approximation of $F$, clearly results in fewer arithmetic operations than using $F$ itself for a fixed $h$ and decreasing $\nu$. Moreover, the difference increases with increasing problem size though we have not shown this here. 
Next we will study a problem that has a flow that is not uni-directional. We consider flow over a backwards facing step, see Figure 3.

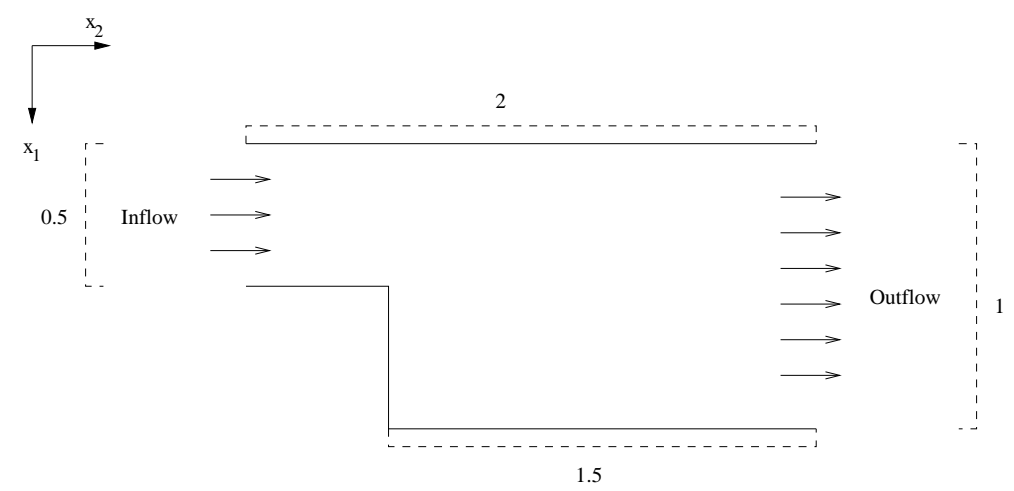

Figure 3: The computational domain.

We start by solving a Stokes problem (2) and use this solution as initial guess $\mathbf{u}^{(0)}$ in the Picard iteration. By denoting the unknowns in the region before the step by $\mathbf{u}_{1}$ and those after the step by $\mathbf{u}_{2}$ we obtain a partitioned system of equations

$$
\left(\begin{array}{cccc}
F_{1} & F_{12} & B_{1}^{T} & B_{12}^{T} \\
F_{21} & F_{2} & B_{21}^{T} & B_{2}^{T} \\
B_{1} & B_{12} & 0 & 0 \\
B_{21} & B_{2} & 0 & 0
\end{array}\right)\left(\begin{array}{l}
\mathbf{u}_{1} \\
\mathbf{u}_{2} \\
\mathbf{p}_{1} \\
\mathbf{p}_{2}
\end{array}\right)=\left(\begin{array}{c}
\mathbf{f}_{1} \\
\mathbf{f}_{2} \\
0 \\
0
\end{array}\right)
$$

to solve at each Picard iteration. In order to have a fast solver for the semi-circulant approximation to $F$ we omit the coupling between the two subdomains and employ

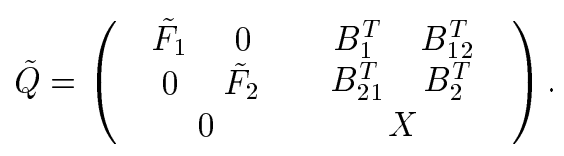

We use zero Dirichlet boundary conditions for the velocities at solid walls. At inflow we set $u_{1}\left(x_{1}, 0\right)=0, u_{2}\left(x_{1}, 0\right)=x_{1}\left(0.5-x_{1}\right)$ and at outflow $u_{1}\left(x_{1}, 2\right)=0$, $u_{2}\left(x_{1}, 2\right)=0.5^{3} x_{1}\left(1-x_{1}\right)$. We comment that the outflow boundary condition at $x_{2}=2$ is not particularly realistic in terms of channel flow. To obtain a more realistic physical problem we could either have extended the channel and/or applied a 'natural' outflow boundary condition. However in either case the flow in the solution domain would become more unidirectional and so we would expect our preconditioner to perform essentially as in our first example. We have specifically chosen the boundary condition given here to induce more variability in flow direction i.e. more variability in the coefficients of the advection terms. As convergence criterion 
for the linear iterations we use

$$
\frac{\left\|\left(\begin{array}{l}
\mathbf{f} \\
0
\end{array}\right)-\left(\begin{array}{cc}
F & B^{T} \\
B & 0
\end{array}\right)\left(\begin{array}{l}
\mathbf{u} \\
\mathbf{p}
\end{array}\right)^{k}\right\|_{2}}{\left\|\left(\begin{array}{l}
\mathbf{f} \\
0
\end{array}\right)\right\|_{2}} \leq 10^{-3}
$$

and for the Picard iteration

$$
\max \left(\mathbf{u}^{(i)}-\mathbf{u}^{(i-1)}\right) \leq 10^{-6}
$$

In Tables 6-7 we display the number of nonlinear iterations and the average number of GMRES-iterations per nonlinear iteration required for convergence for different values of $h$ and $\nu$.

\begin{tabular}{|c|c|c|}
\hline$h$ & $1 / 21$ & $1 / 41$ \\
\hline$\nu=1$ & $6(2)$ & $7(2)$ \\
$\nu=1 / 10$ & $8(3)$ & $9(3)$ \\
$\nu=1 / 100$ & $9(4)$ & $11(4)$ \\
$\nu=1 / 200$ & $9(5)$ & $12(5)$ \\
\hline
\end{tabular}

Table 6: Average number of preconditioned GMRES iterations per nonlinear iteration using the preconditioner $\mathcal{Q}$. Within parenthesis we display the number of Picard iterations.

\begin{tabular}{|c|c|c|}
\hline$h$ & $1 / 21$ & $1 / 41$ \\
\hline$\nu=1$ & $23(2)$ & $33(2)$ \\
$\nu=1 / 10$ & $23(3)$ & $33(3)$ \\
$\nu=1 / 100$ & $23(4)$ & $33(4)$ \\
$\nu=1 / 200$ & $24(5)$ & $36(5)$ \\
\hline
\end{tabular}

Table 7: Average number of preconditioned GMRES iterations per nonlinear iteration using the preconditioner $\mathcal{Q}_{x_{2}}$. Within parenthesis we display the number of Picard iterations.

\section{Conclusions}

We have presented a preconditioner for the linearised incompressible Navier-Stokes problem (the Oseen problem) based on semi-circulant approximation of advectiondiffusion and the $B F B T$-preconditioner of Elman for the pressure Schur complement block.

We have indicated theoretically how use of our preconditioner in the favourable situation of a constant wind (giving rise to constant coefficients) and periodic boundary conditions will lead to convergence (termination) of a Krylov subspace method in just 2 iterations independently of mesh size and Reynolds number. We then show numerical results for two less ideal test problems, one with constant 
advective wind and Dirichlet boundary conditions and one employing Picard iteration for the full Navier-Stokes equations in an expanding channel geometry with Dirichlet boundary conditions which were specifically chosen to induce variable flow direction. In both cases convergence is seen to be independent of the Reynolds number and only mildly dependent on the mesh size so long as there is adequate mesh to resolve the solution.

We conclude that this is an effective preconditioning technique for the incompressible Navier-Stokes equations.

\section{References}

[1] F. Brezzi and M. Fortin, Mixed and Hybrid Finite Element Methods, SpringerVerlag, New York (1991).

[2] T. F. Chan, An optimal circulant preconditioner for Toeplitz systems, SIAM J. Sci. Stat. Comput., 9 (1988), pp. 766-771.

[3] I. S. Duff, A. M. Erisman and J. K. Reid, Direct methods for sparse matrices, Oxford University Press, Oxford (1986).

[4] H. C. Elman, Multigrid and Krylov Subspace Methods for the Discrete Stokes Equations, Int. J. Numer. Methods Fluids, 22 (1996), pp. 755-770.

[5] H. C. Elman, Preconditioning for the steady-state Navier-Stokes equations with low viscosity, SIAM J. Sci. Comput., 20 (1999), pp. 1299-1316.

[6] H. C. Elman, and D. J. Silvester, Fast Nonsymmetric Iterations and Preconditioning for Navier-Stokes Equations SIAM J. Sci. Comput., 17 (1996), pp. $33-46$.

[7] H. E. Elman, D. J. Silvester and A. J. Wathen, Iterative methods for problems in Computational Fluid Dynamics, in 'Iterative Methods in Scientific Computing', Eds. R.H. Chan, T.F. Chan and G.H. Golub, Springer-Verlag, Singapore, pp. 271-327.

[8] R. Freund, and N. Nachtigal, QMR: A quasi-minimal residual method for nonHermitian linear systems, Numer. Math., 60 (1991), pp. 315-339.

[9] G. H. Golub, and A. J. Wathen, An iteration for indefinite systems and its application to the Navier-Stokes equations, SIAM J. Sci. Comput., 19(2) (1998), pp. 530-539.

[10] P. M. Gresho and R. L. Sani, On pressure boundary conditions for the incompressible Navier-Stokes equations, Int. J. Numer. Methods Fluids, 7 (1987), pp. 1111-1145.

[11] F.H. Harlow and J.E. Welsh, Numerical calculation of time-dependent viscous incompressible flow of fluid with free surfaces, Phys. Fluids, 8 (1965), pp. 21812189. 
[12] L. Hemmingsson, A semi-circulant preconditioner for the convection-diffusion equations, Numer. Math., 81 (1998), pp. 211-249.

[13] S. Holmgren, and K. Otto, A framework for polynomial preconditioners based on fast transforms I: Theory, BIT, 38 (1998), pp. 544-559.

[14] T. Y. Hou and B. T. R. Wetton, Second-order convergence of a projection scheme for the incompressible Navier-Stokes equations with boundaries, SIAM J. Numer. Anal., 30 (1993), pp. 609-629.

[15] O. A. Karakashian, On a Galerkin-Lagrange multiplier method for the stationary Navier-Stokes equations, SIAM J. Numer. Anal., 19 (1982), pp. 909-923.

[16] C. van Loan, Computational Frameworks for the fast Fourier transform, SIAM, Philadelphia 1992.

[17] M. F. Murphy, G.H. Golub and A. J. Wathen, A note on preconditioning for indefinite linear systems, Report No. 99/07, Oxford University Computing Laboratory, accepted for publication in SIAM J. Sci. Comput., 1999.

[18] M. F. Murphy and A. J. Wathen, On preconditioning for the Oseen equations, University of Bristol, Department of Mathematics Report AM-95-07, (1995).

[19] S.V. Patankar, Numerical Heat Transfer and Fluid Flow, Hemisphere, Washington (1980).

[20] A. Ramage and A. J. Wathen, Iterative solution techniques for the Stokes and Navier-Stokes equations, Int. J. Numer. Meths. Fluids 19 (1994), pp. 67-83.

[21] Y. Saad and M. H. Schultz, GMRES: A generalized minimal residual algorithm for solving nonsymmetric linear systems, SIAM J. Sci. Stat. Comput., 7 (1986), pp. 856-869.

[22] D. J. Silvester and A. J. Wathen, Fast iterative solution of stabilised Stokes systems Part II: Using general block preconditioners, SIAM J. Numer Anal. 31 (1994), pp. 1352-1367.

[23] M. Suri and R. Stenberg, Mixed hp finite element methods for problems in elasticity and Stokes flow, Numer. Math., 72 (1996), pp. 367-390.

[24] H. A. van der Vorst, Bi-CGSTAB: A fast and smoothly converging variant of Bi-CG for the solution of nonsymmetric linear systems SIAM J. Sci. Stat. Comput. 13 (1992), pp. 631-644. 